\title{
Rate of Correct Use of Folic Acid Supplementation Among Pregnant Women - Beijing Municipality, China, 2017-2019
}

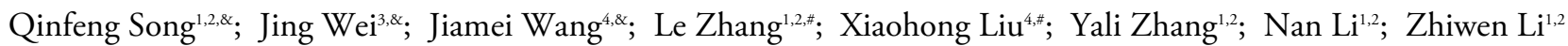

\section{Summary \\ What is already known on this topic? \\ Neural tube defects can be effectively prevented by folic acid supplementation. However, compliance with the recommendations for supplementation is still low in China. \\ What is added by this report? \\ This study investigated the rate of correct use of folic acid supplementation and its risk factors among pregnant women in Beijing. Women who took folic acid correctly only comprised less than $50 \%$ of the total, possibly due to unexpected pregnancy. \\ What are the implications for public health practice? \\ Given the low rate in Beijing, an area with high health literacy, the rate may also be low in other areas in China. Regulations for mandatory fortification of food with folic acid are recommended in China.}

Some studies have reported unsatisfactory compliance with folic acid supplementation recommendations in China. In a study encompassing several regions of China, only $38.4 \%$ women initiated folic acid supplementation at least 3 months before conception (whether the intake after conception was sufficient was unclear) (1), which was significantly less than the target of $70 \%$ set by the National Health Commission (NHC) of the People's Republic of China (2). Beijing has similar socioeconomic characteristics to various developed countries and may reflect future conditions in China. Based on the baseline survey of a cohort study, this study aimed to determine the rate of correct use of folic acid consumption (sufficient not only before but also after conception) among pregnant women in Beijing and to understand its risk factors. A structured questionnaire was administered to 3,988 pregnant women; $97.2 \%$ of the women reported taking folic acid supplements (folic acid alone or multivitamin tablets with folic acid), but only $24.2 \%$ were compliant with the recommended dose and duration of folic acid supplementation. Unexpected pregnancy was a major risk factor associated with the correct use of folic acid. The evidence from this study suggests that regulations for mandatory fortification of food with folic acid are required in China.

Data were obtained from a baseline survey of an ongoing prospective cohort study. The participants included 3,988 pregnant women at or before 16 weeks of gestation when enrolling through convenience sampling (selected from a prenatal health checkup and the maternity school) at the Haidian Maternal and Child Health Hospital, China, between August 2017 and November 2019. Among them, 132 women were excluded because their folic acid intake was unclear; therefore, 3,856 women were included in the final analysis. Data collected via the three-part structured questionnaire were entered twice into EpiData software (version 3.1, EpiData Association, Odense, Denmark). This study was approved by the institutional review board, and all participants provided written informed consent.

In pregnant women, the correct use of folic acid could be achieved by the intake of no less than 24 capsules of folic acid per month, from 3 months before to 3 months after conception. The rate of correct use of folic acid supplementation was calculated as the percentage of pregnant women who consumed an adequate dose of folic acid for over the period delineated above. Crude odd ratios (cORs) and adjusted odd ratios (aORs) were calculated to evaluate risk levels of factors. Chi-square and Fisher's tests were used to compare categorical variables, and logistic regression was performed with adjustment for confounding factors including survey year, age at recruitment, education, pregnancy planning, and parity. $P$-values $<0.05$ were considered statistically significant. SPSS Statistics software (version 24.0; IBM Corp., Armonk, NY, USA) was used for data analysis.

The mean age of the participants was 31 years (range: $21-49$ years); $73.5 \%$ had a bachelor's degree or higher, $82.0 \%$ lived in urban areas, $79.1 \%$ were nulliparas, $29.3 \%$ were unexpected pregnancies, and $97.2 \%$ took folic acid supplements. Only $24.2 \%$ of 
women started taking folic acid supplements at the recommended dosage 3 months before conception (Table 1).

The results of single-factor analysis (Table 2) showed that the percentage of women taking folic acid correctly differed significantly by age, education, pregnancy planning, and parity (all $P<0.05$ ). Correct use of folic acid supplementation was observed in $4.1 \%$ of unexpected pregnancies, far lower than planned pregnancies (32.5\%). In addition, unexpected pregnancy occurred in $36.9 \%$ (992 out of 2,685) of women who took folic acid incorrectly. Pregnant women with planned pregnancies [aOR: 10.31;95\% confidence interval (CI): 7.44-14.29] and nulliparas (aOR: 1.48 ; 95\% CI: $1.15-1.90$ ) were more likely to take folic acid correctly (Table 3). Between 2017 and 2019, the rate of correct use of folic acid supplementation significantly increased (aOR: 1.42; 95\% CI: 1.12-1.80). Pregnant women aged $\leq 25$ years were more likely to take folic acid incorrectly (aOR: 0.43; 95\% CI: 0.26-0.72).

\section{DISCUSSION}

The results of this study revealed that among pregnant women in a knowledge-intensive area of
Beijing, 97.2\% took folic acid supplementation, but only $24.2 \%$ took supplementation correctly. If using the less stringent United States Preventive Services Task Force standard (at least 24 capsules of folic acid per month from 1 month before to 3 months after conception), the rate of correct use of folic acid supplementation just marginally increased to $40.3 \%$. The rate in Beijing was similar to that in Western countries (42.2\%) (3) but was still very low. Importantly, this rate likely reflected future conditions in China. Although the rate of correct use of folic acid supplementation increased over time, it was still significantly below the NHC target $(70 \%)$.

In this study, $29.3 \%$ of pregnant women were unexpected pregnancies. Unexpected pregnancies occurred in more than a third of women who took folic acid incorrectly and were associated with a significantly increased risk of incorrect use of folic acid. Unexpected pregnancies were common in China, ranging from $20.6 \%$ to $39.6 \%$ of all (4-6). Women with unexpected pregnancies often miss the best time for folic acid supplementation, which makes it one of the major risk factors for the correct use of folic acid throughout the world. Furthermore, the one-child policy was abolished in China in 2016, which may increase the likelihood of unexpected pregnancies over

TABLE 1. Folic acid supplementation among pregnant women in Haidian District, 2017-2019.

\begin{tabular}{|c|c|c|}
\hline Variable & Participants (number) & Percentage (\%) \\
\hline \multicolumn{3}{|l|}{ Whether or not folic acid was taken } \\
\hline Yes & 3,748 & 97.2 \\
\hline No & 108 & 2.8 \\
\hline \multicolumn{3}{|l|}{ When folic acid supplementation began } \\
\hline 3 months before conception & 1,090 & 29.1 \\
\hline 2 months before conception & 350 & 9.3 \\
\hline 1 month before conception & 431 & 11.5 \\
\hline Within 1 month after conception & 749 & 20.0 \\
\hline Later than 1 month after conception & 1,030 & 27.5 \\
\hline Unknown & 98 & 2.6 \\
\hline \multicolumn{3}{|l|}{ Frequency of folic acid intake ${ }^{*}$} \\
\hline 24 capsules or more per month & 2,833 & 75.6 \\
\hline 15-23 capsules per month & 610 & 16.3 \\
\hline Fewer than 15 capsules per month & 129 & 3.4 \\
\hline Unknown & 176 & 4.7 \\
\hline \multicolumn{3}{|l|}{ Correct use of folic acid } \\
\hline Yes & 880 & 24.2 \\
\hline No & 2,763 & 75.8 \\
\hline
\end{tabular}

* Among pregnant women who had taken folic acid. 
TABLE 2. Single-factor analysis of risk factors of correct use of folic acid among pregnant women in Haidian District, 2017-2019.

\begin{tabular}{|c|c|c|c|}
\hline Variable & Correct use, n (\%) & Incorrect use , n (\%) & $P$ \\
\hline \multicolumn{4}{|l|}{ Year } \\
\hline 2017 & $194(22.3)$ & $675(77.7)$ & \multirow{3}{*}{0.006} \\
\hline 2018 & $438(23.2)$ & $1,454(76.8)$ & \\
\hline 2019 & $248(28.1)$ & $634(71.9)$ & \\
\hline \multicolumn{4}{|l|}{ Age, years } \\
\hline$\leq 25$ & $28(11.2)$ & $223(88.8)$ & \multirow{4}{*}{$<0.001$} \\
\hline $26-30$ & $474(24.9)$ & $1,426(75.1)$ & \\
\hline $31-35$ & $298(27.6)$ & $781(72.4)$ & \\
\hline$>35$ & $75(18.8)$ & $325(81.2)$ & \\
\hline \multicolumn{4}{|l|}{ Education } \\
\hline High school or lower & $45(16.5)$ & $228(83.5)$ & \multirow{3}{*}{0.005} \\
\hline Undergraduate & $568(24.2)$ & $1,778(75.8)$ & \\
\hline Master's degree or higher & $265(26.0)$ & $755(74.0)$ & \\
\hline \multicolumn{4}{|l|}{ Residence } \\
\hline City area & $698(24.1)$ & 2,195 (75.9) & \multirow{3}{*}{0.786} \\
\hline Near suburbs & $157(25.2)$ & $466(74.8)$ & \\
\hline Far outskirts & $2(18.2)$ & $9(81.8)$ & \\
\hline \multicolumn{4}{|l|}{ Unexpected pregnancy } \\
\hline Yes & $42(4.1)$ & $992(95.9)$ & \multirow{2}{*}{$<0.001$} \\
\hline No & $816(32.5)$ & $1,693(67.5)$ & \\
\hline \multicolumn{4}{|l|}{ Parity } \\
\hline Nulliparas & $734(26.4)$ & $2,050(73.6)$ & \multirow{2}{*}{$<0.001$} \\
\hline Multiparas & $117(16.0)$ & $614(84.0)$ & \\
\hline
\end{tabular}

time, leading to a lower rate of correct use of folic acid supplementation. In this study, nulliparas were significantly more likely to take folic acid correctly than multiparas. Compared to nulliparas, multiparas pay less attention to folic acid supplementation because of their previous experience with pregnancy. In 2019, more than $60 \%$ of births in China were of a second child and above (7). This proportion and the proportion of unexpected pregnancies are likely to increase due to the recently announced three-child policy. As a result, the rate of correct use of folic acid supplementation may decrease, which will delay attainment of the NHC target. Compared to the effects of unexpected pregnancies and parity, the impact of education on correct folic acid supplementation was found to be limited in the higheducated sample.

The US Food and Drug Administration was the first public health body to mandate folic acid supplementation in 1997, which proved to be a successful strategy to reduce birth defects (8). At present, 88 countries and regions have mandated folate fortification in cereal grains to ensure adequate folic acid consumption in women of childbearing age (9). In a district of Beijing, the prevalence of neural tube defects (NTDs) at $\geq 28$ weeks of gestation (a commonly used indicator) was $73.0 \%$ lower than the prevalence of NTDs throughout pregnancy $(11 / 10,000$ population) because severe defects often lead to termination of pregnancy before 28 weeks of gestation (10). With such a severe situation, folic acid supplementation is important for NTDs prevention at all times in China. Despite the large number of implemented programs, the rate of correct use of folic acid supplementation was still far below the NHC target, even in the most developed Chinese city. Therefore, besides improved health literacy in underdeveloped areas, mandatory folate fortification supplementation is urgently required all over China.

This study was subject to at least two limitations: convenience sampling provides a less representative sample (overrepresenting nulliparas), and self-reported 
TABLE 3. Logistic regression analysis of risk factors of correct use of folic acid among pregnant women in Haidian District, 2017-2019.

\begin{tabular}{|c|c|c|c|c|}
\hline Variable & cOR $(95 \% \mathrm{Cl})$ & $P$ & aOR $(95 \% \mathrm{Cl})$ & $P$ \\
\hline \multicolumn{5}{|l|}{ Year } \\
\hline 2017 & 1 & & 1 & \\
\hline 2018 & $1.05(0.87-1.27)$ & 0.632 & $1.12(0.91-1.38)$ & 0.280 \\
\hline 2019 & $1.36(1.10-1.69)$ & 0.005 & $1.42(1.12-1.80)$ & 0.004 \\
\hline \multicolumn{5}{|l|}{ Age, years } \\
\hline$\leq 25$ & $0.54(0.34-0.87)$ & 0.011 & $0.43(0.26-0.72)$ & 0.002 \\
\hline $26-30$ & $1.44(1.10-1.89)$ & 0.008 & $0.98(0.71-1.36)$ & 0.917 \\
\hline $31-35$ & $1.65(1.24-2.20)$ & 0.001 & $1.16(0.84-1.60)$ & 0.368 \\
\hline$>35$ & 1 & & 1 & \\
\hline \multicolumn{5}{|l|}{ Education } \\
\hline High school or lower & 1 & & 1 & \\
\hline Undergraduate & $1.62(1.16-2.26)$ & 0.005 & $1.51(1.02-2.25)$ & 0.039 \\
\hline Master's degree or higher & $1.78(1.25-2.52)$ & $<0.001$ & $1.46(0.97-2.20)$ & 0.072 \\
\hline \multicolumn{5}{|l|}{ Unexpected pregnancy } \\
\hline No & $11.38(8.27-15.68)$ & $<0.001$ & $10.31(7.44-14.29)$ & $<0.001$ \\
\hline Yes & 1 & & 1 & \\
\hline \multicolumn{5}{|l|}{ Parity } \\
\hline Nulliparas & $1.88(1.52-2.33)$ & $<0.001$ & $1.48(1.15-1.90)$ & 0.002 \\
\hline Multiparas & 1 & & 1 & \\
\hline
\end{tabular}

Abbreviations: $c \mathrm{OR}=$ crude odd ratio; $\mathrm{aOR}=$ adjusted odd ratio; $\mathrm{Cl}=$ confidence interval.

questionnaires may inevitably cause information bias. Nevertheless, based on the low rate of correct use of folic acid supplementation in Beijing, an area with high health literacy, it can be speculated that folic acid supplementation and voluntary fortification of folic acid might not be effective for preventing NTDs in China. Regulations for mandatory fortification of food such as cereal grains with folic acid might be necessary to prevent NTDs.

Conflicts of Interest: No conflict of interest exits in the submission of this manuscript.

Acknowledgements: Families participating in this study; the workers at the Haidian Maternal and Child Health Hospital.

Funding: The National Natural Science Foundation of China (No. 81973056, and 81673177).

doi: $10.46234 / \mathrm{ccdcw} 2021.194$

\# Corresponding authors: Le Zhang, zhangle@bjmu.edu.cn; Xiaohong Liu,13522099566@163.com.

\footnotetext{
Institute of Reproductive and Child Health, Peking University/Key Laboratory of Reproductive Health, National Health Commission of the People's Republic of China, Beijing, China; ${ }^{2}$ Department of Epidemiology and Biostatistics, School of Public Health, Peking University, Beijing, China; ${ }^{3}$ Cancer Center of Shanxi Bethune Hospital, Shanxi Academy of Medical Science, Taiyuan, Shanxi,
}

China; ${ }^{4}$ Haidian Maternal and Child Health Hospital, Beijing, China. \& Joint first authors.

Submitted: August 11, 2021; Accepted: September 06, 2021

\section{REFERENCES}

1. Li Q, Wang YY, Guo YM, Zhou H, Wang XB, Wang QM, et al. Folic acid supplementation and the association between maternal airborne particulate matter exposure and preterm delivery: a national birth cohort study in China. Environ Health Perspect 2020;128(12):127010. http://dx.doi.org/10.1289/ehp6386.

2. National Health Commission of the People's Republic of China. Notice of the issuance of "2010 management protocol for the prevention project of neural tube defects with folic acid supplementation" by the ministry ofhealthgeneral office.2010.http://www.nhc.gov.cn/zwgk/wtwj/ 201304/13c65b34969d4bbba88d67bd66614303.shtml. [2021-5-25]. (In Chinese).

3. Fulford B, Macklon N, Boivin J. Mental models of pregnancy may explain low adherence to folic acid supplementation guidelines: a crosssectional international survey. Eur J Obstet Gynecol Reprod Biol 2014;176:99 - 103. http://dx.doi.org/10.1016/j.ejogrb.2014.02.011.

4. Xu YN, Wu YQ, Lv X, You Y. Analysis on folic acid intake of pregnant women and its influencing factors in Sichuan Province. Mod Prev Med 2013;40(16):3009-11, 3014. https://t.cnki.net/kcms/detail?v=3uoqIh G8C46NmWw7YpEsKL-WhGHP2RH_vXCTWQUdTnWU9jdt Fcy3UfA2fg9tri2VxXYobAEjqJFMqywnDdXO2EQQ9xP2estW\&unip latform=NZKPT. (In Chinese).

5. Li WJ, Yang WF, Zeng LX, Mu LH, Li YS, Li K, et al. KAP investigation on birth defects of childbearing women and its influencing factors in Yan'an City. Chin J Woman Child Health Res 2017;28(7): 
797-801. https://d.wanfangdata.com.cn/periodical/gwyx-fybj2017 07015. (In Chinese).

6. Yang S, Liu YL, Dang WS, Zhang XJ. Statue of folic acid intake of the urban pregnant women of Shanxi province and its influence factors. Chin J Fam Plan 2018;26(1):1019-23. https://d.wanfangdata.com.cn/ periodical/ChlQZXJpb2RpY2FsQ0hJTmV3UzIwMjEwODE4EhJ6Z2 poc314enoyMDE4MTEwMDIaCGdsZWhtNGJp. (In Chinese).

7. Department of Planning, Development and Information Technology. Statistical communiqué of the People's Republic of China on the national health care and service development in 2019. 2020. http:// www.nhc.gov.cn/guihuaxxs/s10748/202006/ebfe31f24cc145b198dd73 0603ec4442.shtml. [2020-10-20]. (In Chinese).
8. Centers for Disease Control \& Prevention. Spina bifida and anencephaly before and after folic acid mandate-United States, 1995-1996 and 1999-2000. MMWR Morb Mortal Wkly Rep 2004;53(17):362, 364-5. https://www.jstor.org/stable/23315440.

9. Fortification Initiative Food. Mandatory cereal grain fortification legislation- July 2021. 2021. https://www.ffinetwork.org/globalprogress. [2021-7-30].

10. Yu JR, Jin L, Xiao LH, Jin L. Prevalence of neural tube defects and its association with monitoring time in Tongzhou district, Beijing. J Peking Univ (Health Sci) 2015;47(6):1042 - 5. http://dx.doi.org/10. 3969/j.issn.1671-167X.2015.06.030. (In Chinese). 